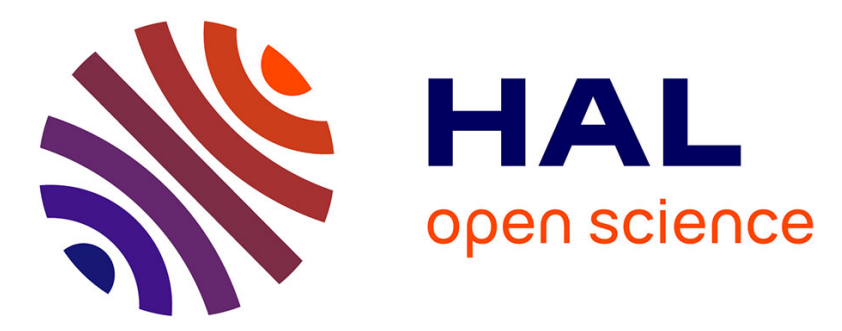

\title{
Singular ordinary differential equations homogeneous of degree 0 near a codimension 2 set
}

Didier Bresch, Benoit Desjardins, Emmanuel Grenier

\section{To cite this version:}

Didier Bresch, Benoit Desjardins, Emmanuel Grenier. Singular ordinary differential equations homogeneous of degree 0 near a codimension 2 set. 2009. hal-00408619

\section{HAL Id: hal-00408619 \\ https://hal.science/hal-00408619}

Preprint submitted on 31 Jul 2009

HAL is a multi-disciplinary open access archive for the deposit and dissemination of scientific research documents, whether they are published or not. The documents may come from teaching and research institutions in France or abroad, or from public or private research centers.
L'archive ouverte pluridisciplinaire HAL, est destinée au dépôt et à la diffusion de documents scientifiques de niveau recherche, publiés ou non, émanant des établissements d'enseignement et de recherche français ou étrangers, des laboratoires publics ou privés. 


\title{
Singular ordinary differential equations homogeneous of degree 0 near a codimension 2 set
}

\author{
Didier Bresch ${ }^{1}$, Benoît Desjardins ${ }^{2}$, Emmanuel Grenier ${ }^{3}$
}

\begin{abstract}
This note deals with an example of class of ordinary differential equations which are singular near a codimension 2 set, with an homogeneous singularity, of degree 0. Under some structural assumptions, we prove that for almost all initial data there exists a unique global solution and study the evolution of the Lebesgue measure of a transported set of initial data.
\end{abstract}

Keywords : Singular ODE's, co-dimension 2 singularity, global existence and uniqueness

AMS subject classification : 47305, 37N10, 35A05, 74H35.

\section{Introduction}

This note deals with a simple example of a class of ordinary differential equations which are singular on a manifold of codimension 2, the singularity being homogeneous of degree 0 near this singularity.

Let $H$ be an Hilbert space. Let $\Pi$ denotes the orthogonal projection on a given plane $P$. Let $x_{h}=\Pi x$ and let $x_{v}=x-\Pi x$. Note that $x=x_{h}+x_{v}$. By a slight abuse of notation we shall say that $x=\left(x_{h}, x_{v}\right), x_{h}$ and $x_{v}$ referring to the "horizontal" and "vertical" components of $x$. We then identify the plane $P$ with $\mathbb{C}$ by choosing arbitrarily a basis on $P$. This allows to use polar coordinates on $P$ and thus to define the modulus $r$ and argument $\theta$ of $x_{h}$, and to use the notation $x_{h}=r \exp (i \theta)$. Then

Let $\phi$ be a smooth function defined on $H \times \mathbb{S}^{1}$ ( $\mathbb{S}^{1}$ being the unit circle).

$$
\dot{x}=\phi\left(x, \frac{x_{h}}{\left|x_{h}\right|}\right)
$$

is a dynamical system, singular on $P^{\perp}$, orthogonal of $P$, which is of codimension 2. Moreover, this system is homogeneous of degree 0 in any direction orthogonal to $P^{\perp}$.

Under some assumptions on $\phi$, we show global existence and uniqueness of solution for almost every initial data. Note that we only take care of the behavior of $t \mapsto x(t)$ near $P^{\perp}$ and by a slight abuse of language we shall say that a solution is global if it does not reach the singularity $P^{\perp}$ in finite time.

\footnotetext{
${ }^{1}$ Didier.bresch@univ-savoie.fr, LAMA, UMR5127 CNRS, Université de Savoie, 73376 Le Bourget du lac, France

${ }^{2}$ Benoit.Desjardins@mines.org, ENS Ulm, D.M.A., 45 rue d'Ulm, 75230 Paris cedex 05, France, and Modélisation Mesures et Applications S.A., 66 avenue des Champs Elysées, 75008 Paris, France

3 egrenier@umpa.ens-lyon.fr, U.M.P.A., École Normale Supérieure de Lyon, 46, allée d'Italie, 69364 Lyon Cedex 07, France.
} 


\section{Motivation}

The toy model (1) mimics phenomena occurring in the low Mach number limit problem for non-isentropic flows in a periodic box for instance. Such flows are described through a velocity field $u$, a density $\rho$ implicitly given by a state law depending on the entropy $S$ and the pressure $p$, see [4]). After some change of variables, namely introducing $q$ defined by $p=\bar{p} e^{\varepsilon q}$ with $\bar{p}$ a prescribed constant and $\varepsilon$ the low Mach number, the adimensionnalized system reads

$$
\begin{gathered}
a\left(\partial_{t} q+u \cdot \nabla q\right)+\frac{1}{\varepsilon} \operatorname{div} u=0, \\
r\left(\partial_{t} u+u \cdot \nabla u\right)+\frac{1}{\varepsilon} \nabla p=0, \\
\partial_{t} S+u \cdot \nabla S=0,
\end{gathered}
$$

where $a$ and $r$ are two smooth functions of $S$ and $\varepsilon q$. The singular limit consists in letting the Mach number $\varepsilon$ go to 0 . In the ill prepared-data case, namely when the initial data do not satisfy the condition

$$
\operatorname{div} u^{0}=0, \quad \nabla p^{0}=0 .
$$

an oscillatory limit with changing eigenvalues occurs. Indeed the eigenvalues and the spectrum of the singular operator $\mathcal{A}$ where

$$
\mathcal{A}=\left(\begin{array}{cc}
0 & a^{-1} \operatorname{div} \\
r^{-1} \nabla & 0
\end{array}\right)
$$

will depend on the solution itself. This leads to complex problem, since eigenvalues may cross. The wave equation, related to the operator $\mathcal{A}$, may also be written in this case

$$
\varepsilon \partial_{t t} \psi-\operatorname{div}\left(S^{-1} \nabla \psi\right)=0
$$

where $S$ is the entropy quantity, see [4] for more details.

In the very special case of only one spatial dimension, the limit can be both calculated completely and justified, see [4]. In the multi-dimensional case the formal calculation of the extra term in the limit, which once again involves the spectral decomposition of the fast operator, assumes that the spectrum of that fast operator is simple and non-resonant, see [3] for the viscous case and [4] for the inviscid one. For certain finite-dimension al truncations of the equations those assumptions can be shown to be generic and to ensure convergence to the limit equations. This has been done in the paper [4].

The main difficulty in the general study is to prove, after defining appropriate infinite dimension measures, that for almost all initial data, the limit flow does not meet double eigenvalues and crosses the resonance set transversally. Difficulties occur since the flow is singular across the resonant set which may be shown to define a codimension 2 set. This explain the necessity to study ODEs of the form (1). Our result will be used to treat oscillatory limits with changing eigenvalues and particularly the low Mach number limit in a forthcoming paper [2]. Note that another type of singular ordinary differential equation occuring in fluid mechanics has been studied recently, see for instance [1]. 


\section{Setup and main result}

\subsection{Notations.}

Let us first introduce some notations. We define $\psi\left(x_{h}, x_{v}, \theta\right)$ as the argument of $\Pi \phi\left(\left(x_{h}, x_{v}\right), \exp (i \theta)\right)$, and $\tilde{\psi}\left(x_{h}, x_{v}, \theta\right)$ its modulus in such a way that

$$
\Pi \phi\left(\left(x_{h}, x_{v}\right), \exp (i \theta)\right)=\tilde{\psi}\left(x_{h}, x_{v}, \theta\right) \exp \left(i \psi\left(x_{h}, x_{v}, \theta\right)\right) .
$$

Structural properties. We will assume that there exists an integer $N_{0}>0$ and $N_{0}$ smooth functions of $x_{v}, \Theta_{1}\left(x_{h}, x_{v}\right), \ldots, \Theta_{N_{0}}\left(x_{h}, x_{v}\right)$, satisfying the following properties :

(H1) For all $x=\left(x_{h}, x_{v}\right) \in H$, the equation in $\theta$

$$
\psi\left(x_{h}, x_{v}, \theta\right) \in \theta+\pi \mathbb{Z}
$$

has exactly $N_{0}$ solutions $\Theta_{1}\left(x_{h}, x_{v}\right) \ldots \Theta_{N_{0}}\left(x_{h}, x_{v}\right)$.

(H2) For every $j$, the following sign condition holds for all $x=\left(x_{h}, x_{v}\right) \in H$

$$
\partial_{\theta} \psi\left(x_{h}, x_{v}, \Theta_{j}\left(x_{h}, x_{v}\right)\right)<1 .
$$

Note that this implies that the solutions $\Theta_{j}$ are all simple.

(H3) $\tilde{\psi}$ does not vanish.

Note that $(\mathrm{H} 1)$ implies that $\left\{\left(\tau \exp \left(i \Theta_{j}\left(0, x_{v}\right)\right), x_{v}\right), \tau>0\right\}$ is a trajectory for

$$
\dot{x}_{h}=\Pi \phi\left(\left(0, x_{v}\right), \frac{x_{h}}{\left\|x_{h}\right\|}\right) .
$$

This trajectory goes to the singularity or leaves it, depending on its orientation. In particular for some initial data we reach the singularity in finite time. The flow is not defined everywhere and we can only get almost everywhere results.

\subsection{Main result and consequences}

The main result of this paper is :

Theorem 3.1 Stable and unstable manifolds.

Let us assume that (H1), (H2) and (H3) hold true. Let $x_{0} \in P^{\perp}$ and let $\rho>0$. There exists a finite number of manifolds $V_{k}$, of codimension 1 , with boundary $\Sigma$, such that :

- For any initial data $x_{1}$ in one of the manifolds $V_{k}$, the corresponding solution of (9) reaches $\Sigma$ in finite time (in the past or in the future).

- For any initial data $x_{1}$ in $B\left(x_{0}, \rho\right)$ outside all these manifold $V_{k}$, the corresponding solution of (9) reaches the boundary of $B\left(x_{0}, \rho\right)$ before $\Sigma$. 
With this result one can define the flow $\Psi(t)$ of this equation, flow which is defined everywhere except on the manifolds $V_{k}$. Let $A$ be some set of initial data. We now want to bound the measure of $\Psi(t) A$ in terms of the measure of $A$. This of course depends on the measure of $H$, which is not canonical. In order to fix the ideas we will restrict ourselves to a finite dimensional space $H$, with the Lebesgue measure $\mu$, in order to prove

Theorem 3.2 Control of the divergence.

Let $x_{0} \in P^{\perp}$. Let $\rho>0$. There exists a constant $C_{0}(t)$ such that for any borelian set $A$ of $H$,

$$
\mu\left(\Psi(t)\left(A \cap B\left(x_{0}, \rho\right)\right)\right) \leq C_{0}(t) \mu\left(A \cap B\left(x_{0}, \rho\right)\right),
$$

for any $t>0$.

The outline of the paper is as follows. First we study the simplified equation

$$
\dot{x}=\phi\left(\frac{x_{h}}{\left|x_{h}\right|}\right)
$$

and then extend it by perturbation arguments to equations of the general form (1). More precisely, in Section 4, we will study trajectories near the singular set. The last section concerns measure type estimate of a transported set by the flow which is defined everywhere except on the manifolds $V_{k}$ of codimension one.

\section{Study of systems of the form (9)}

We first prove Theorem 2.1 in the particular case of systems of the form (9). Note that (9) is a two dimensional dynamical system, which takes places in $x_{h}+P$. Changing the notations, (9) is of the form

$$
\dot{y}=\phi\left(\frac{y}{\|y\|}\right)
$$

where $y \in \mathbb{C}$. First we turn to polar coordinates, define $y=r \exp (i \theta)$ and make the change of time defined by $d \tau / d t=1 / r$ to get

$$
\begin{aligned}
\frac{d r}{d \tau} & =r \tilde{\psi}(\theta) \cos (\psi(\theta)-\theta) \\
\frac{d \theta}{d \tau} & =\tilde{\psi}(\theta) \sin (\psi(\theta)-\theta) .
\end{aligned}
$$

Note that (11) does not involve $r$, which greatly simplifies the analysis of (9). Note also that $\tilde{\psi}$ is always positive. Therefore the dynamics of (11) is given by Assumption (H1). There exists $N_{0}$ fixed points $\Theta_{j}\left(x_{v}\right)$ which are stable provided

$$
\left(\psi^{\prime}\left(\Theta_{j}\right)-1\right) \cos \left(\psi\left(\Theta_{j}\right)-\Theta_{j}\right)<0
$$


and unstable if $\left(\psi^{\prime}\left(\Theta_{j}\right)-1\right) \cos \left(\psi\left(\Theta_{j}\right)-\Theta_{j}\right)>0$ (the null case being ruled out by $(\mathrm{H} 2)$ ).

Moreover the $\Theta_{j}$ are the only fixed points of (11) and the dynamics of solutions $t \mapsto \theta(t)$ of (11) is very simple $: t \mapsto \theta(t)$ goes in a monotonic way from some $\Theta_{j}\left(x_{v}\right)$ (unstable equilibrium, limit value as $\tau$ goes to $-\infty$ ) to $\Theta_{j-1}\left(x_{v}\right)$ or $\Theta_{j+1}\left(x_{v}\right)$ as $\tau$ goes to $+\infty$ (stable equilibria). All the solutions of (11) are global in the $\tau$ variable and go from an unstable $\Theta_{j}$ to a close stable one.

It then remains to solve (10). The behavior depends on the sign of

$$
\tilde{\psi}(\theta) \cos (\psi(\theta)-\theta) .
$$

As $\tilde{\psi}(\theta)>0$ and as $\psi^{\prime}\left(\Theta_{j}\right)<1, \tilde{\psi}(\theta) \cos (\psi(\theta)-\theta)$ is positive if $\Theta_{j}$ is stable and negative if $\Theta_{j}$ is unstable.

Therefore solutions of (9) are global (except if $\theta$ constantly equals some of the $\Theta_{j}$ where the solution goes to the singularity in finite time in the future or in the past), and are asymptotic in $+\infty$ to some stable $\Theta_{j}$ and in $-\infty$ to some instable $\Theta_{j}$.

The phase portrait can be described as follows :

- There exists $N_{0}$ particular solutions which are straight lines, going to or coming from the origin in finite positive or negative times.

- All the other trajectories are global in time and are asymptotic to two of the particular solutions as time goes to $+\infty$ or $-\infty$.

Theorem 2.1 is then straightforward.

Note that Hypothesis (H2) is crucial. If we assume $\psi^{\prime}\left(\Theta^{\prime}\right)>1$, then the conclusion is completely changed : all the trajectories come from the singularity and go back to the singularity in finite time, except for $\theta=\Theta_{j}$. In this case, almost all the trajectories blow up in finite time.

\section{$5 \quad$ Trajectories near the singular set}

In this section we will describe the behavior of solutions near the singular set. Let $x_{0}=\left(0, x_{v}^{0}\right)$ be a point of $P^{\perp}$. Locally the geometry of the flow is described by the angles $\Theta_{j}\left(x_{v}^{0}\right)$ which split the space into angular sectors

$$
\Omega_{j}=\left\{\Theta_{j}\left(x_{v}^{0}\right)<\theta<\Theta_{j+1}\left(x_{v}^{0}\right)\right\} .
$$

If there were no $x_{h}$ dependence of the flow, the angles $\Omega_{j}$ would be invariant under the flow as in the previous section. This is not the case here and we have to be more precise in the spatial description.

\subsection{Domain decomposition.}

Let $\alpha>0$ and $\eta>0$ be small. Then by continuity there exists angles $\theta_{j}^{+}$ and $\theta_{j}^{-}$such that

$$
\left|\tilde{\psi}\left(x_{h}, x_{v}, \theta\right) \sin \left(\psi\left(x_{h}, x_{v}, \theta\right)-\theta\right)\right| \geq \alpha
$$


when $\left|x_{h}\right|+\left|x_{v}-x_{v}^{0}\right|<2 \eta$ and $\Theta_{j}\left(x_{v}^{0}\right)<\theta_{j}^{+}<\theta<\theta_{j+1}^{-}<\Theta_{j+1}\left(x_{v}^{0}\right)$.

Let us now introduce the following sets, for $\varepsilon$ chosen later on with $\varepsilon<\eta$ :

$$
\begin{gathered}
\Omega_{j}(\varepsilon, \eta)=\left\{\left(x_{h}, x_{v}, \theta\right), \quad|\quad| x_{h}|<\varepsilon, \quad| x_{v}-x_{v}^{0} \mid<\eta, \quad \theta_{j}^{-}<\theta<\theta_{j}^{+}\right\} \\
\Sigma_{j}(\varepsilon, \eta)=\left\{\left(x_{h}, x_{v}, \theta\right), \quad|\quad| x_{h}|<\varepsilon, \quad| x_{v}-x_{v}^{0} \mid<\eta, \quad \theta_{j}^{+}<\theta<\theta_{j+1}^{-}\right\}
\end{gathered}
$$

and

$$
\Omega_{v}(\varepsilon, \eta)=\left\{\left(x_{h}, x_{v}, \theta\right), \quad|\quad| x_{h}|<\varepsilon, \quad| x_{v}-x_{v}^{0} \mid<\eta\right\} .
$$

Note that $\Omega_{v}(\varepsilon, \eta)$ is the union of the $\Omega_{j}(\varepsilon, \eta)$ and $\Sigma_{j}(\varepsilon, \eta)$ and of their boundaries. The $\Omega_{j}(\varepsilon, \eta)$ will be the neighborhood of the stable and unstable manifolds.

\subsection{Study of the trajectory.}

Let us assume to fix the ideas that $\Theta_{j}$ is unstable and $\Theta_{j+1}$ is stable (the discussion is similar if the stabilities are interchanged). Let $x(t)$ be a trajectory with $x(0) \in \Sigma_{j}(\varepsilon, \eta / 2)$. We claim

Claim : If $\varepsilon$ is small enough, the trajectory $x(t)$ is contained in $\Omega_{j}(\eta, \eta)$ for $t<t_{-}$and is contained in $\Omega_{j+1}(\eta, \eta)$ for $t>t_{+}$. Moreover, for $|t|$ large enough, it exits $\Omega_{v}(\eta)$.

Proof of the claim : Let $x(t)$ be a solution of (1), such that $x(0)=\left(x_{h}(0), x_{v}(0)\right)$ with $x_{h}(0)=r(0) \exp (i \theta(0))$. Using polar coordinates and introducing again the change of time variable, we get

$$
\dot{x}_{v}=r(1-\Pi) \phi\left(\left(x_{h}, x_{v}\right), \frac{x_{h}}{\left\|x_{h}\right\|}\right),
$$

with

$$
\begin{gathered}
\dot{r}=r \tilde{\psi}\left(x_{h}, x_{v}, \theta\right) \cos \left(\psi\left(x_{h}, x_{v}, \theta\right)-\theta\right) \\
\dot{\theta}=\tilde{\psi}\left(x_{h}, x_{v}, \theta\right) \sin \left(\psi\left(x_{h}, x_{v}, \theta\right)-\theta\right) .
\end{gathered}
$$

Let $] a, b\left[\right.$ be the maximal time interval containing 0 such that $x(t) \in \Omega_{v}(\eta)$ for $t \in] a, b\left[\right.$. By definition of $\theta_{j}$ and $\theta_{j+1}, \theta(t)$ is increasing as long as it belongs to $\Omega_{v}(\eta, \eta)-\Omega_{j}(\eta, \eta)-\Omega_{j+1}(\eta, \eta)$. Let $] a_{1}, b_{1}$ [ the maximum time interval containing 0 such that $\theta_{j}<\theta(t)<\theta_{j+1}$. Of course $a \leq a_{1}<b_{1} \leq b$.

If $a<a_{1}$ then for $t=a_{1}, x(t) \in \Omega_{j} \cap \Omega_{v}$ and therefore $x(t) \in \Omega_{j} \cap \Omega_{v}$ for any $a<t<a_{1}$. Similarly, if $b>b_{1}$ then for any $b_{1}<t<b, x(t) \in \Omega_{j+1} \cap \Omega_{v}$.

Note that, using (15) and (12), we can bound $b_{1}-a_{1}$, by

$$
b_{1}-a_{1} \leq \frac{\theta_{3}-\theta_{2}}{\alpha} .
$$

This implies that $b_{1}$ and $-a_{1}$ are less than $\left(\theta_{3}-\theta_{2}\right) / \alpha$ Moreover

$$
\frac{d}{d \tau} \log r=\tilde{\psi}\left(x_{h}, x_{v}, \theta\right) \cos \left(\psi\left(x_{h}, x_{v}, \theta\right)-\theta\right)
$$


Note that on $\Omega_{v}, \tilde{\psi}$ is bounded, by some constant $C_{0}$. Hence for $a_{1}<\tau<b_{1}$, integrating (17) with respect to $\tau$ and using (16), we get

$$
\exp \left(-C_{0} \alpha^{-1}\left(\theta_{3}-\theta_{2}\right)\right) \leq \frac{r(\tau)}{R_{0}(x)} \leq \exp \left(C_{0} \alpha^{-1}\left(\theta_{3}-\theta_{2}\right)\right) .
$$

Therefore if

$$
\varepsilon<\frac{\eta}{1+2 C_{0}} \exp \left(-2 C_{0} \alpha^{-1}\left(\theta_{3}-\theta_{2}\right)\right),
$$

$r(\tau)$ remains smaller than $\eta$ for $a_{1}<\tau<b_{1}$. Hence the solution can not leave $\Omega_{v}$ at $\tau=a_{1}$ or $\tau=b_{1}$ at the boundary $\left|x_{h}\right|=\eta$.

Now using (13), we see that as, using (18)-(19), $r(\tau)$ remains smaller than $\eta / 2 C_{0}$, the trajectory can not leave $\Omega_{v}$ at its horizontal boundary. Therefore the trajectory goes from $\Omega_{j}(\eta)$ to $\Omega_{j+1}(\eta)$, which ends the proof of the claim.

Bounds on $r(t)$ : Let us go back to the genuine time $t$. On $\Omega_{j}(\eta)$ and $\Omega_{j+1}(\eta)$, provided $\alpha$ is small enough, $\left|\cos \left(\psi\left(x_{h}, x_{v}, \theta\right)-\theta\right)\right|$ is larger than $1 / 2$. Therefore

$$
\frac{d}{d t}\left|x_{h}(t)\right|^{2}=2 r(t) \dot{r}(t)
$$

is bounded away from 0 by $r(t) \min \tilde{\psi} / 2$. Therefore, as long as $r(t)$ remains in $\Omega_{v}$, for $t>b_{1}$ we have

$$
r\left(b_{1}\right)+\gamma_{1}\left(t-b_{1}\right) \leq r(t) \leq r\left(b_{1}\right)+\gamma_{2}\left(t-b_{1}\right)
$$

for some non negative constants $\gamma_{1}, \gamma_{2}$. A similar result is true for $t<a_{1}$.

Dynamics on $\Omega_{j}$ : As $\dot{r}>0$ we change time to get

$$
\begin{gathered}
\dot{x}_{v}=\frac{r}{\cos \left(\psi\left(x_{h}, x_{v}, \theta\right)-\theta\right)}(1-\Pi) \phi\left(\left(x_{h}, x_{v}\right), \frac{x_{h}}{\left\|x_{h}\right\|}\right), \\
\dot{r}=r, \\
\dot{\theta}=\tan \left(\psi\left(x_{h}, x_{v}, \theta\right)-\theta\right) .
\end{gathered}
$$

Note that $(20,21,22)$ is a standard dynamical system, studied near the equilibrium set $\left\{\left(x_{v}, 0, \Theta_{j}\right) \mid x_{v} \in P^{\perp}\right\}$ which appears to be unstable. It therefore admits an unstable manifold, which is precisely the manifold $V_{j}$.

\section{Measure of a transported set}

This section is divided in three parts. The first one concerns a control of the integral of the divergence along a trajectory $x(t)$ passing through a point $x_{0}$ in terms of its minimum distance $R_{0}(x)$ to the singularity. The second part concerns the local intergrability of $\log \left(R_{0}^{-1}\right)$ that will be used in the third part to prove Theorem 2.2 through the bound established in the first part. 
Control of the divergence. Let us first bound the divergence of our vector field. This divergence $D$ equals

$$
D=\frac{1}{r}\left(\cos \left(\psi\left(x_{h}, x_{v}, \theta\right)-\theta\right)\left(\partial_{\theta} \psi-1\right) \tilde{\psi}+\partial_{\theta} \tilde{\psi} \sin \left(\left(\psi\left(x_{h}, x_{v}, \theta\right)-\theta\right)\right)+D_{1}\right.
$$

where $D_{1}$ is a sum of terms which are bounded as $r$ goes to 0 . If $\alpha$ and $\eta$ are small enough (see the domain decomposition part for their definitions), the sine is nearly 0 and much smaller than the cosine, hence $D$ has the sign of $\cos \left(\Theta_{j}-\theta\right)$ and, as $\partial_{\theta} \psi \neq 1$ and $\tilde{\psi}$ is bounded away from $0, D$ is bounded in modulus by

$$
|D| \leq \frac{c_{0}}{2 r}
$$

provided $\alpha$ is small enough.

Let us now evaluate the integral of the divergence along a trajectory. Let $x(t)$ with $t_{1}<t<t_{2}$ be a part of trajectory included in $\Omega_{j+1}$. Note that $r(t)$ is increasing and

$$
r(t) \geq r\left(t_{1}\right)+C_{0}\left(t-t_{1}\right)
$$

for some constant $C_{0}$ depending only on $\tilde{\psi}$. Therefore,

$$
\begin{gathered}
\int_{t_{1}}^{t_{2}} \frac{1}{r(t)} d t \leq \int_{t_{1}}^{t_{2}} \frac{1}{r\left(t_{1}\right)+C_{0}\left(t-t_{1}\right)} d t=\frac{1}{C_{0}} \log \left(\frac{r\left(t_{1}\right)+C_{0}\left(t_{2}-t_{1}\right)}{r\left(t_{1}\right)}\right) \\
\leq \frac{1}{C_{0}} \log \left(\frac{\eta}{R_{0}(x)}\right)
\end{gathered}
$$

since $r\left(t_{2}\right)+C_{0}\left(t_{2}-t_{1}\right) \leq \eta$. A similar result is true for parts of trajectories included in $\Omega_{j}$. Note that the integral behaves like $\log \left(1 / R_{0}(x)\right)$.

Local integrability and minimum distance to the singularity set. It remains to provide a local integrability property related to $\log \left(R_{0}^{-1}\right)$, where $R_{0}^{-1}$ denotes the minimum distance between the singularity and a trajectory $x(t)$ passing trough $x_{0}$. Let us look at the formulation $(13,14,15)$, and let us study a trajectory $x(t)$. Up to a shift in time, we may assume that $x(t)$ reaches $R_{0}$ at $t=0$. Let us study $x(t)$ when $t$ becomes negative and large. First $x(t)$ enters $\Omega_{j}(\varepsilon, \eta)$ at some time $t_{1}<0$ with $\left|t_{1}\right|=O(1)$ as we consider sequences of trajectories with corresponding $R_{0}$ which go to 0 .

For $t<t_{1}$, when $d\left(x(t), P^{\perp}\right)=\delta(\delta$ being fixed) then, using (14) and integrating from 0 to $t, t$ is of order $C_{1} \log \left(\delta / R_{0}\right)$. Since the trajectory $x(t)$ approaches $V_{j}$ with an exponential speed, we get that $d\left(x(t), V_{j}\right)$ is of order $C_{2}\left(R_{0} / \delta\right)^{\gamma}$ for some positive constants $C_{2}$ and $\gamma$.

Therefore the measure of

$\left\{x_{0} \in \Omega_{j}(\varepsilon, \eta) \mid d\left(x(t), P^{\perp}\right)=\delta\right.$, the trajectory $x(t)$ passes at a distance smaller than $\left.r_{0}\right\}$ is of order $C_{3}\left(r_{0} / \delta\right)^{\gamma}$ for some positive constants $C_{3}$ and $\gamma$. Thus

$$
\mu\left(x: R_{0}(x) \leq r_{0} \text { and } d\left(x, P^{\perp}\right)=\delta\right) \leq C\left(r_{0} / \delta\right)^{\gamma} .
$$


Hence $\log \left(R_{0}^{-1}\right)$ is locally integrable.

Bound of the measure of a transported set. Let $F\left(t_{0}, t_{1}\right)$ denotes the resolvent of the flow, which is defined outside the manifolds $V_{j}$, and thus defined almost everywhere. Let $x_{0} \in P^{\perp}$. Let $\rho>0$, then for any measurable set $A$,

$$
\begin{gathered}
\mu\left(F\left(t_{0}, t_{1}\right)\left(A \cap B\left(x_{0}, \rho\right)\right)=\mu(A) \exp \left(\int_{t_{0}}^{t_{1}} \int_{F\left(t_{0}, \tau\right)\left(A \cap B\left(x_{0}, \rho\right)\right)} D(\tau, x) d \tau d x\right)\right. \\
=\mu\left(A \cap B\left(x_{0}, \rho\right)\right) \exp \left(\int_{A \cap B\left(x_{0}, \rho\right)} \int_{t_{0}}^{t_{1}} D\left(\tau, F\left(t_{0}, \tau\right) x\right) d \tau d x\right) \\
\leq \mu\left(A \cap B\left(x_{0}, \rho\right)\right) \exp \left(C_{0}^{-1} \int_{A \cap B\left(x_{0}, \rho\right)} \log \left(\eta R_{0}^{-1}(x)\right) d x\right) \leq C \mu\left(A \cap B\left(x_{0}, \rho\right)\right)
\end{gathered}
$$

for some constant $C$ since $\log \left(R_{0}^{-1}\right)$ is locally integrable. This ends the proof of Theorem 2.2.

Acknowledgments. The first author would like to thank the French ANR Project "MathOcean" (2008-2011) managed by D. LANNES.

\section{Références}

[1] S. Bianchini, L.V. Spinolo. Invariant manifolds for a singular ordinary differential equations. Submitted (2008).

[2] D. Bresch, B. Desjardins, E. Grenier. Oscillatory Limits with Changing Eigenvalues. In preparation (2009).

[3] D. Bresch, B. Desjardins, E. Grenier, C.-K. Lin. Low Mach number limit of viscous polytropic flows : formal asymptotics in the periodic case. Stud. Appl. Math. 109 (2002), no. 2, 125-149.

[4] G. MÉTIvier, S. Schochet. Averaging theorems for conservative systems and the weakly compressible Euler equations. J. Differential Equations 187 (2003), no. 1, 106-183. 